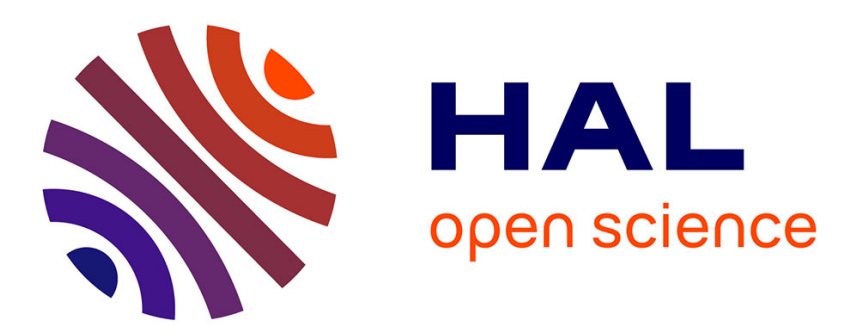

\title{
Un mode de production inédit au Paléolithique moyen dans l'industrie du niveau 6e du Pucheuil (Seine-Maritime)
}

Anne Delagnes

\section{- To cite this version:}

Anne Delagnes. Un mode de production inédit au Paléolithique moyen dans l'industrie du niveau 6e du Pucheuil (Seine-Maritime). PALEO : Revue d'Archéologie Préhistorique, 1993, 5, pp.111-120. halshs-00447541

\author{
HAL Id: halshs-00447541 \\ https://shs.hal.science/halshs-00447541
}

Submitted on 15 Jan 2010

HAL is a multi-disciplinary open access archive for the deposit and dissemination of scientific research documents, whether they are published or not. The documents may come from teaching and research institutions in France or abroad, or from public or private research centers.
L'archive ouverte pluridisciplinaire $\mathbf{H A L}$, est destinée au dépôt et à la diffusion de documents scientifiques de niveau recherche, publiés ou non, émanant des établissements d'enseignement et de recherche français ou étrangers, des laboratoires publics ou privés. 


\title{
UN MODE DE PRODUCTION INEDIT AU PALEOLITHIQUE MOYEN DANS L'INDUSTRIE DU NIVEAU 6e DU PUCHEUIL (SEINE-MARITIME)
}

\begin{abstract}
Anne Delagnes *
Résumé :

L'une des séries Paléolithique moyen du gisement du Pucheuil se caractérise par une production secondaire originale, réalisée à partir des sousproduits de la chaîne opératoire principale (Levallois). Ses spécificités techniques ainsi que la chronologie des opérations de débitage sont successivement décrites.
\end{abstract}

Abstract.

One of the Middle Paleolithic series from the site of Le Pucheuil contains an original secondary production, realized on the by-products of the main reduction process (based on the Levallois system). Its technical characieristics and the chronology of the debitage are here described in succession.

Le gisement du Pucheuil (Saint-Saëns, SeineMaritime) a fait l'objet de deux fouilles de sauvetage (sous la direction d'Anne Ropars) au cours des hivers 1990 et 1991 dans le cadre de l'aménagement de l'autoroute A28. Ces deux campagnes de sauvetage ont permis la mise au jour d'une importante séquence loessique comprenant plusieurs niveaux archéologiques distincts, corrélés à différents stades du Saalien (études géochronologiques en cours sous la direction de J.P. Lautridou). A la base des dépôts, un bief à silex (silex du Crétacé) a fourni aux hommes préhistoriques toute la matière première nécessaire à leurs besoins en termes de production lithique.

Parmi les différents niveaux inclus dans cette séquence, le niveau 6e a livré une industrie particulièrement abondante et très pcu perturbće. Typologiquement, cette série se rattache a l'ensemble des industries Levalloisiennes du Nord de la France caractérisées par de très faibles taux d'outils retouchés (Commont, 1913 ; Bordes et Bourgon, 1951 ; Bordes, 1953).

L'intérêt de ce matériel est surtout d'ordre technique, dans la mesure où il a donné lieu à de nombreux remontages allant jusqu'à la reconstitution presque complète de plusieurs blocs de silex. Ces ensembles remontés permettent d'appréhender de façon très précise les liens techniques entre les différents modes de production qui coexistent dans cette série. La chaîne opératoire principale est fondée sur le débitage Levallois, qui repose lui-même essentiellement (mais pas exclusivement) sur une production récurrente unipolaire convergente menant à l'obtention de pointes et d'éclats Levallois triangulaires (la même méthode de débitage a été identifiée dans le gisement moustérien d'Houppeville, situé à une trentaine de kilomètres : Vallin, 1992).

Plusieurs chaînes općratoires secondaires viennent se greffer à la chaîne opératoire Levallois. L'une d'elle est régie par un mode de production original, qui n'a jusqu'à présent pas d'équivalent connu dans le Palćolithique inféricur et moyen d'Europe occidentale (sur la base des données bibliographiques). La description de ce mode de production, que j'ai dénommé en raison de son caractère inédit : débitage de type Le Pucheuil, est l'objet du présent article.

\section{DEFINITION}

Le débitage de type Le Pucheuil se définit comme un système de production d'éclats récurrents unidirectionnels, superposés les uns aux autres de façon à ce que chaque éclat ôte entièrement le négatif de l'éclat précédent.

\section{FICHE TECHNIQUE}

- Technique de percussion : percuteur dur

- Méthode de débitage : production récurrente reposant sur la réalisation d'une série d'éclats (de 2 à 8) produits à partir du même plan de frappe et selon la même direction de façon à ce que chaque éclat soit un peu plus envahissant que le précédent dont il emporte entièrement le négatif (fig.1). 
- Conception volumétrique : le débitage s'effectue à partir de supports présentant deux surfaces sécantes. Les éclats sont produits aux dépens de la surface la plus plane, l'autre surface étant utilisée comme plan de frappe. Au début du débitage le plan de fracturation des éclats (selon l'axe de débitage) est quasiment parallèle à la surface exploitcé. Progressivement il est de plus en plus tangentiel et tend à devenir quasiment perpendiculaire à cette surface en fin d'exploitation (fig.1). Cette conception volumétrique est totalement différente de celles qui caractérisent les systèmes de production jusqu'alors définis pour le Palćolithique inférieur et moyen (Boëda, Geneste et Meignen, 1990 ; Boëda, 1991).

- Supports de départ : tous les sous-produits de la chaîne opératoire principale (Levallois), d'une épaisseur supérieure à $15-20 \mathrm{~mm}$, et principalement :

* éclats d'entame et éclats corticaux appartenant aux phases de configuration et d'initialisation des nucléus Levallois,

* fragments, débris et éclats issus d'accidents de débitage lors des phases d'exploitation des nucléus Levallois,

* nucléus Levallois (après abandon),

constituent les potentiels supports de ce mode de production (fig.2).

Caractères morphométriques et morphologiques des éclats (fig. 3 ) : ils sont très spécifiques et homogènes. Morphométriquement ce sont des éclats de petites dimensions dont la largeur est supérieure à la longueur (selon l'axe de débitage). Sur un plan morphologique (et morphotechnique):

* En vue de face ils se caractérisent par un talon très large (équivalent à la largeur des éclats), le plus souvent en V, opposé à un tranchant distal tout aussi large et dont la délinéation est sub-rectiligne ou en arc de cercle. Ce tranchant n'est recoupé par aucune arête : il est donc parfaitement lisse sur toute sa longueur.

* En vue de profil : ils se singularisent par une concavité proximale très marquée (créée par le négatif de l'éclat antérieur) et par une silhouctte cambrée. Le profil accuse en effet une nette courbure depuis le sommet du bulbe jusqu'à l'extrémité distale. Le tranchant distal forme ainsi un dièdre déjeté par rapport à l'axe longitudinal de la pièce. Ce dièdre est de section plano-concave, et forme un angle relativement aigu (entre 30 et $40^{\circ}$ ).

* En vue de dessous, ces pièces présentent la morphologie caractéristique des éclats "en aile d'oiseau", avec une large concavité mésiane sur la face supérieure.

\section{CHRONOLOGIE DES OPERATIONS DE DEBITAGE}

La chronologie des opérations appliquées à ce mode de débitage a été reconstituée de façon détaillée. Elle est étayée par de nombreux remontages (tab.1) qui restituent à la fois des séquences partielles du débitage de type Le Pucheuil et des séquences quasiment complètes (fig.4), inscrites dans des blocs dont la plupart des éléments ont pu être raccordés.

\begin{tabular}{|l|c|c|c|}
\hline & Remontés & Non remontés & Total \\
\hline Nucléus & 19 & 9 & 28 \\
\hline Eclats & 45 & 91 & 136 \\
\hline Eclats premiers & 7 & 24 & 31 \\
\hline
\end{tabular}

Tableau 1 : Décompte des nucléus, éclats et éclats premiers de type Le Pucheuil (le nombre des pièces remontées ne tient pas compte des raccords de pièces fracturées)

\section{- Phase 1 : sélection et configuration des supports}

La sélection des supports employés pour le débitage de type Le Pucheuil s'effectue à partir des sous-produits issus des différentes phases de la chaîne opératoire Levallois. Le débitage de type Le Pucheuil correspond donc dans ce site à une production secondaire, fondée sur le recyclage d'éléments extraits de la production principale. C'est là une stratégie qui, tout en recouvrant des schémas très variés (Geneste, 1991), est très fréquente au Paléolithique moyen.

La configuration des supports est intégrée à leur sélection dans la mesure où les produits sélectionnés présentent des caractères volumétriques qui sont d'emblée conformes aux critères requis pour le débitage. A savoir : une surface plane ou très légèrement convexe opposée à une surface d'aspect plus variable et offrant un angle de frappe assez fermé $\left(60\right.$ à $\left.70^{\circ}\right)$ avec la première.

\section{- Phase 2 : initialisation}

Les phases d'initialisation sont très restreintes et le débitage démarre souvent directement après sélection du support. 
Le plan de frappe n'est pas toujours préalablement préparé. Lorsque tel est le cas, il est soit simplement rectifié, soit aménagé en vue de dégager une proéminence à l'emplacement du point de percussion.

La surface de débitage ne fait l'objet d'aucune préparation particulière. C'est toujours la face la plus plane du support qui est exploitée comme surface de débitage (ex : face inférieure des éclats et fragments d'éclats, ou ancienne surface de débitage des nucléus Levallois). Il s'agit dans tous les cas d'une surface non corticale.

Le débitage commence par la production d'un enlèvement mince et de très petites dimensions. A cause de sa position initiale, il se distingue par l'absence du négatif d'un éclat antérieur en zone proximale ; hormis cela, il possède déjà tous les caractères morphologiques qui caractérisent l'ensemble des produits de type Le Pucheuil. Parmi ces éclats "premiers", les éclats Kombewa sont nombreux étant donné que les surfaces débitées sont très fréquemment des faces inférieures d'éclats.

\section{- Phase 3 : exploitation}

* Aménagement des plans de frappe

L'aménagement des plans de frappe est l'objet d'une certaine flexibilité :

- leur préparation initiale tout comme leur réaménagement en cours de débitage ne sont pas systématiques ;

- les modalités d'aménagement des plans de frappe sont elles-mêmes variables.

Cela transparaît dans la variété des types de talon, qui sont fréquemment dièdres, ou encore facettés, lisses ou corticaux. Cette variété typologique reflète la diversité des moyens employés pour une finalité technique qui est en revanche tout à fait constante : elle repose sur le dégagement d'une proéminence au niveau du point de percussion, en vue d'une meilleure précision du coup. Pour que chaque éclat ôte entièrement le négatif de l'éclat antérieur il faut en effet que son point de percussion soit parfaitement superposé au précédent.

Tour à tour : facettage, mise en place d'un plan de frappe dièdre ou exploitation d'une proéminence préexistante sont mis au service de cette finalité. Cet exemple illustre clairement l'insuffisance, en vue de la compréhension des règles et des options techniques qui régissent le débitage, d'une typologie telle que celle qui est généralement appliquée à l'étude des talons des éclats.
* Exploitation de la surface de débitage.

La surface de débitage est exploitée pour la réalisation d'une série récurrente d'éclats. Tous les éclats sont produits consécutivement, sans aucun réaménagement de la surface de débitage en cours d'exploitation.

La récurrence repose dans ce système sur un principe différent de celui qui préside au débitage Levallois récurrent (cf. Boëda, 1986 : 314) puisque :

- non seulement les caractères de chaque éclat sont directement mis en place par l'éclat précédent,

- mais de plus : chaque éclat récrée pour le suivant des caractères strictement identiques.

Cette forme de récurrence entraîne une forte homogénéité des éclats. Par rapport au déroulement chronologique de la série, des variations dans les caractères des éclats interviennent seulement sur deux points précis :

- Les dimensions (longueur et largeur) des premiers enlèvements connaissent une augmentation croissante du fait que chaque éclat est plus envahissant que le précédent. Elles se stabilisent ensuite : en raison de l'inclinaison progressive du plan de fracturation des éclats, leur longueur est rapidement limitée par l'épaisseur du nucléus.

- On observe aussi une légère modification du profil des pièces : plus le plan de fracturation des éclats s'incline, plus l'angle formé par le négatif de l'enlèvement antérieur et la zone distale (recoupant l'initiale surface de débitage) devient fermé.

Le nombre d'éclats produits consécutivement au sein d'une même série est très variable : le minimum décompté (à partir des ensembles remontés) est de 2, le maximum étant de 8.

L'exploitation volumétrique tout à fait particulière des nucléus constitue une limite à la productivité de ce mode de débitage : lorsque le plan de fracturation des éclats devient perpendiculaire (ou subperpendiculaire) à la surface exploitée, le débitage ne peut se poursuivre car l'angle de frappe est alors trop ouvert (fig. $5: 1$ ).

A ce stade le nucléus peut continuer à être exploité pour la production d'une ou plusieurs autres séries d'éclats, réalisées aussi bien aux dépens de la même face qu'aux dépens de l'autre face. Ces séries sont totalement indépendantes les unes des autres. La plupart des nucléus n'ont toutefois livré qu'une seule série d'éclats ; rares sont ceux qui en ont produit plus de trois. 


\section{- Phase 4 : abandon des nucléus}

Au stade d'abandon, les nucléus (fig.5 et 6) comportent donc les négatifs d'une ou plusieurs séries d'enlèvements. Plus le nombre d'enlèvements par série est important, plus la surface d'éclatement forme un angle obtus avec l'initiale surface de débitage. Sans que l'on puisse estimer précisément (en l'absence de remontages) le nombre d'éclats produits par série, on peut néanmoins par ce biais évaluer de façon relative la productivité d'une série.

La fin d'une séquence de débitage de type Le Pucheuil entraîne l'abandon définitif du nucléus : aucun nucléus n'a été réexploité ou transformé postérieurement à des fins différentes.

\section{- Phase 5 : utilisation}

Les éclats de type Le Pucheuil n'ont fait l'objet d'aucune modification intentionnelle postéricure à leur production : on ne compte pas de pièces retouchées parmi ce groupe d'éclats. Il faut signaler par ailleurs que les pièces retouchées sont extrêmement rares dans l'ensemble de la série du niveau 6e.

En dépit de l'absence de tout aménagement des pièces, un certain nombre d'indices permettent d'envisager que la production de type Le Pucheuil a été pour l'essentiel utilisée sur place :

- nombreux parmi ces éclats sont ceux qui ont donné lieu à des remontages. Plusieurs séries presque complètes d'éclats ont été remontées à partir des nucléus.

- L'homogénéité et la spécificité des éclats semblent indiquer que la mise en place des critères fonctionnels auxquels répond la production est entièrement assurée par la séquence de débitage.

- Le tranchant distal d'une partie des éclats présente des micro-retouches et micro-ébréchures qui pourraient être dues à l'utilisation.

Les éclats de type Le Pucheuil du niveau 6e, tout comme les éclats Levallois d'une autre série du même gisement (cf. Delagnes, 1991 et 1992 : 283295) seraient donc de potentiels outils bien qu'ils n'aient fait l'objet d'aucune transformation postérieure à leur débitage (cf. Bordes, 1970). Une évaluation est actuellement en cours (par S. Beyries) pour déterminer l'état de conservation des microtraces d'utilisation sur ces éclats. Si elle était réalisable, l'analyse tracéologique pourrait permettre de préciser le taux d'utilisation et surtout le mode de fonctionnement de ces pièces.

En complément ou à défaut d'analyse tracéologique, une analyse morphofonctionnelle des éclats de type Le Pucheuil est à prévoir pour tenter d'estimer :

- d'une part les potentialités fonctionnelles du tranchant distal,

- et d'autre part, les modes de fonctionnement possibles de ce tranchant en association avec la concavité formée par le négatif de l'éclat antérieur en partie proximale.

\section{COMMENTAIRES}

Le débitage de type Le Pucheuil conduit à la production en série d'éclats dont la morphologie très particulière n'est pourtant pas du tout exclusive de ce mode de débitage. Il suffit en effet de produire consécutivement deux éclats exactement superposés l'un à l'autre pour obtenir un éclat à "talon en aile d'oiseau" (Tixier, Inizan et Roche, 1980) semblable à ceux du Pucheuil.

L'obtention occasionnelle de tels éclats est assez fréquente à toutes les périodes de la préhistoire et tous contextes confondus : elle est selon les cas soit totalement aléatoire, soit consécutive d'aménagements spécifiques des nucléus, notamment au niveau des plans de frappe. Pour faire reculer un plan de frappe ou pour dégager une proéminence (de type "chapeau de gendarme" par exemple), l'enlèvement de deux éclats superposés est un procédé fréquemment employé.

Au Pucheuil le débitage de ces éclats n'est ni aléatoire ni la résultante d'un procédé d'aménagement des nucléus. Il répond à une production systématisée et finalisée : l'intentionalité se situe très clairement au niveau des produits obtenus et non au niveau des conséquences techniques qui en découlent sur les nucléus.

Cette production secondaire associe un certain nombre de critères quantitatifs et de critères qualitatifs précis, mis en place par une séquence opératoire à la fois courte et rigide, fondée sur la répétivité d'un même geste technique. Une telle répétivité, entraînant la production en série d'éclats à partir du même point de percussion et sans réaménagement de la surface exploitée, est possible techniquement grâce à l'inclinaison graduelle du plan de fracturation des éclats.

Expérimentalement, il apparaît que ce mode de débitage n'exige pas un niveau de technicité très élevé : la principale difficulté réside dans le contrôle de la longueur de chaque éclat, qui doit être court 
(selon son axe de débitage) tout en étant plus envahissant que le précédent. La solution la plus efficace qui soit apparue lors des expérimentations pour résoudre cette difficulté, consiste en une percussion très tangentielle par rapport à la surface débitée.

\section{CONCLUSION}

La reconnaissance et la description du débitage de type Le Pucheuil apporte un élément supplémentaire à l'approche de la variabilité des modes de production du Paléolithique inférieur et moyen. Il n'existe en effet, en l'état actuel de nos connaissances, pas d'équivalent à ce mode de débitage dans les industries du Paléolithique inférieur et moyen d'Europe occidentale. Seule la technique de Setouchi (Aita, Kato et Yamanaka, 1991), dans un tout autre contexte (le Paléolithique supérieur d'ExtrêmeOrient), paraît comparable au débitage de type Le Pucheuil.

L'originalité de ce débitage, qui s'intègre dans une forme d'organisation de la production lithique complexe et structurée, repose sur le contraste entre la forte spécificité de la production répondant à des critères de standardisation assez précis, et le faible investissement technique requis.

\section{BIBLIOGRAPHIE}

AITA, Y., KATO, M. et YAMANAKA, I. 1991. Le remontage des pièces lithiques : une illustration des différentes techniques du Paléolithique supérieur du Japon. In : 25 d'études technologiques en préhistoire: bilan et perspectives. Juan-les-Pins, Editions APDCA, p.255-262.
BOEDA, E. 1991. Approche de la variabilité des sytèmes de production lithique des industries du Paléolithique inférieur et moyen : chronique d'une variabilité attendue. Techniques et Culture, 1991 (janvier-décembre), 17-18, p.37-79.

BOEDA, E., GENESTE, J.M. et MEIGNEN, L. 1990. Identification de chaînes opératoires lithiques du Paléolithique ancien et moyen. Paléo, 1990, 2, p.43-80.

BORDES, F. 1953. Levalloisien et Moustérien. Bulletin de la Société Préhistorique Française, 1953, 50(4), p.226-235.

BORDES, F. 1970. Réflexions sur l'outil au Paléolithique. Bulletin de la Société Préhistorique Française, 1970, 67 (C.R.S.M. n ${ }^{\circ}$ ), p.199-202.

BORDES, F. et BOURGON, M. 1951. Le complexe moustérien : Moustériens, Levalloisien et Tayacien. L'Anthropologie, 1951, 55, p.1-23.

COMMONT, V. 1913. Les hommes contemporains du Renne dans la vallée de la Somme. Amiens : Société des Antiquaires de Picardic, 1913, 646p. (Mémoire de la Société des Antiquaires de Picardie, 37)

DELAGNES, A. 1991. Mise en évidence de deux conceptions différentes de la production lithique au Paléolithique moyen. In : 25 ans d'études technologiques en préhistoire : bilan et perspectives. Juan-les-Pins, Editions APDCA, p.125-137.

DELAGNES, A. 1992. L'organisation de la production lithique au Paléolithique moyen: approche technologique à partir de l'étude des industries de La Chaise-de-Vouthon (Charente). Thèse : Université Paris X - Nanterre, 1992, 386p.

GENESTE, J.M. 1991. Systèmes techniques de production lithique : variations techno-économiques dans les processus de réalisation des outillages paléolithiques. Techniques et Culture, 1991 (janvierdécemble), 17-18, p.1-35.

TIXIER, J., INIZAN, M.L. et ROCHE, H. 1980. Préhistoire de la pierre taillée, 1 : terminologie et technologie. Valbonne : Centre de Recherches et d'Etudes Préhistoriques, 1980, 120p.

VALLIN, L. 1992. Le gisement moustérien d'Houppeville-Les Hautes Terres Sud (SeineMaritime) : étude d'un assemblage lithique en milieu loessique. Revue archéologique de l'Ouest, 1992, 9, p.5-37. 

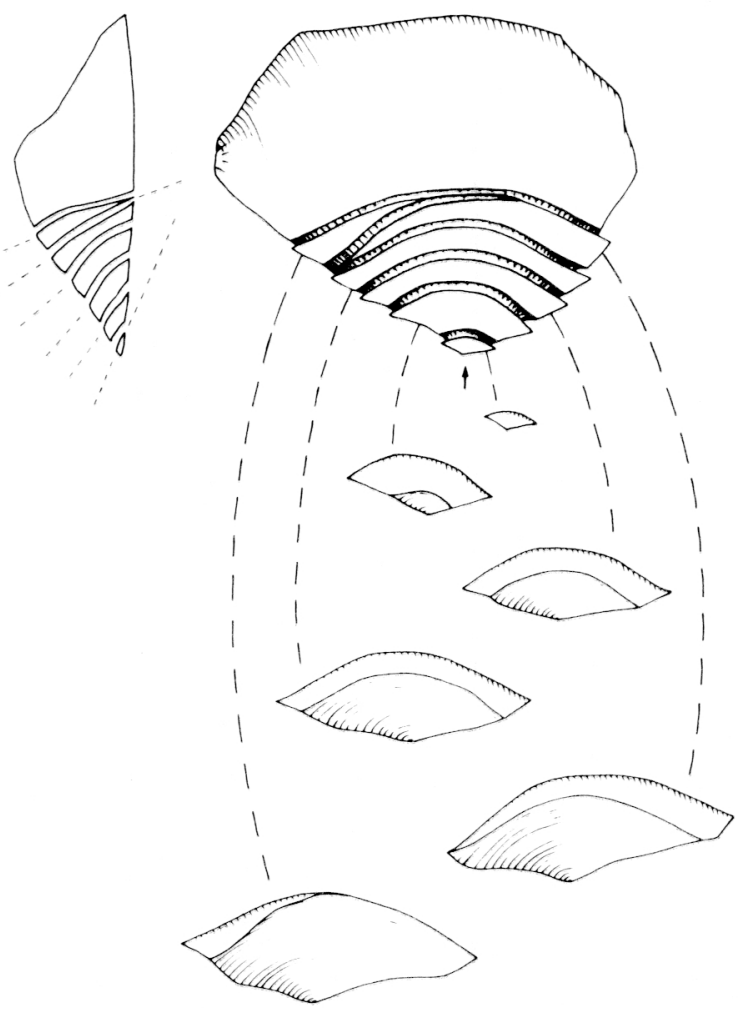

- Figure 1 : Principes techniques et volumétriques du débitage de type Le Pucheuil

\section{Chaîne opératoire}

principale

(Levallois)

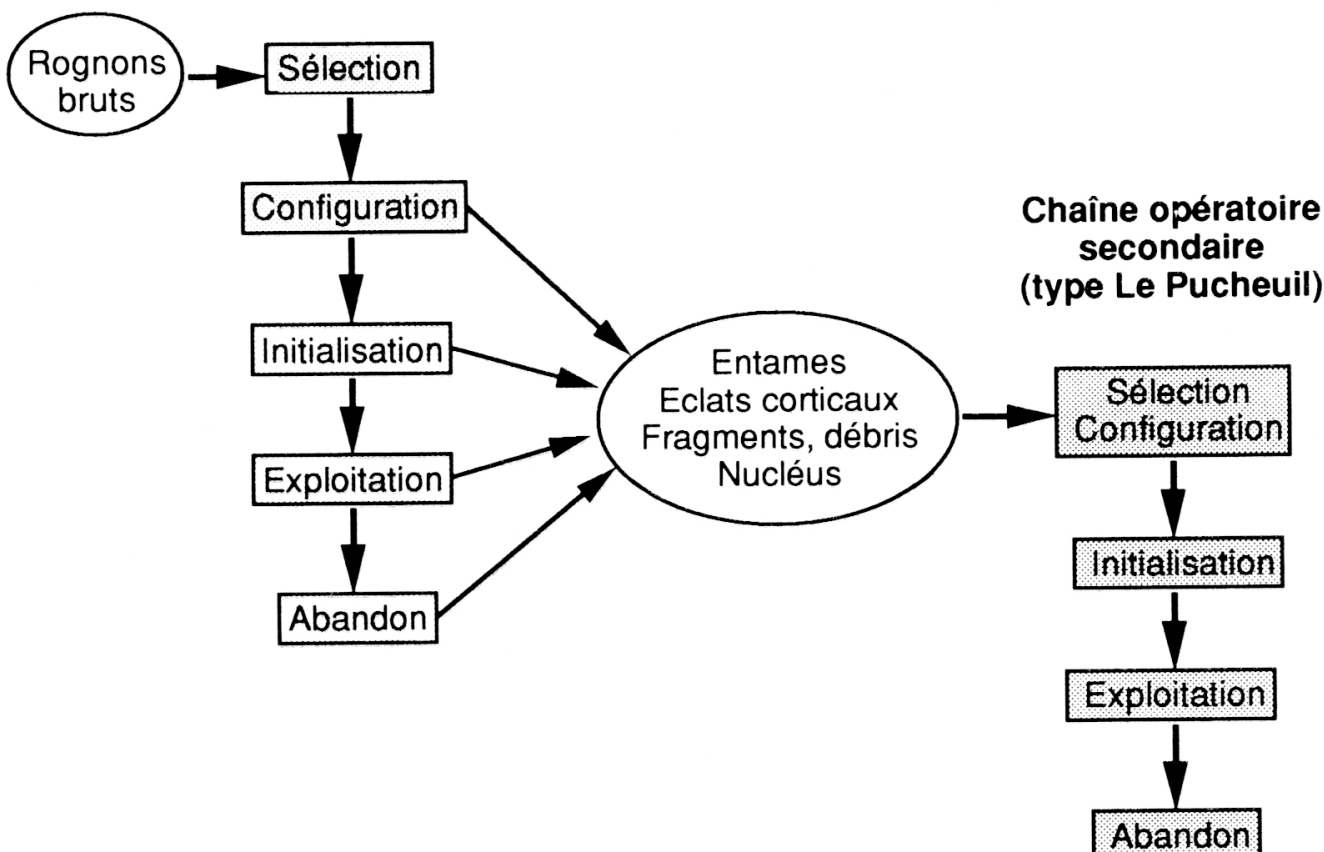

- Figure 2: Les chaînes opératoires (phases de débitage) Levallois et de type Le Pucheuil dans l'industrie du niveau $6 \mathrm{e}$ du Pucheuil 

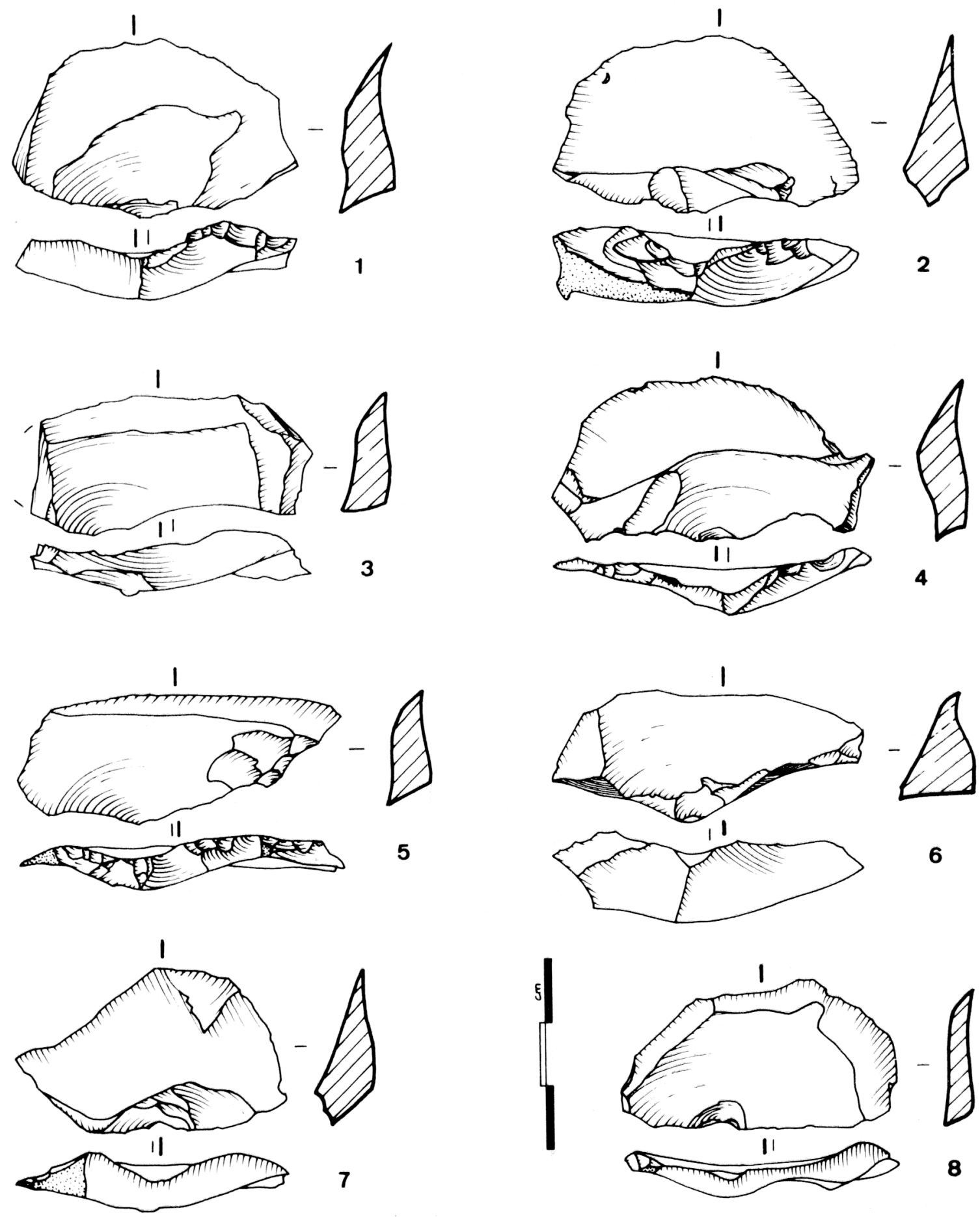

- Figure 3 : Le Pucheuil, niveau 6e : éclats de type Le Pucheuil 

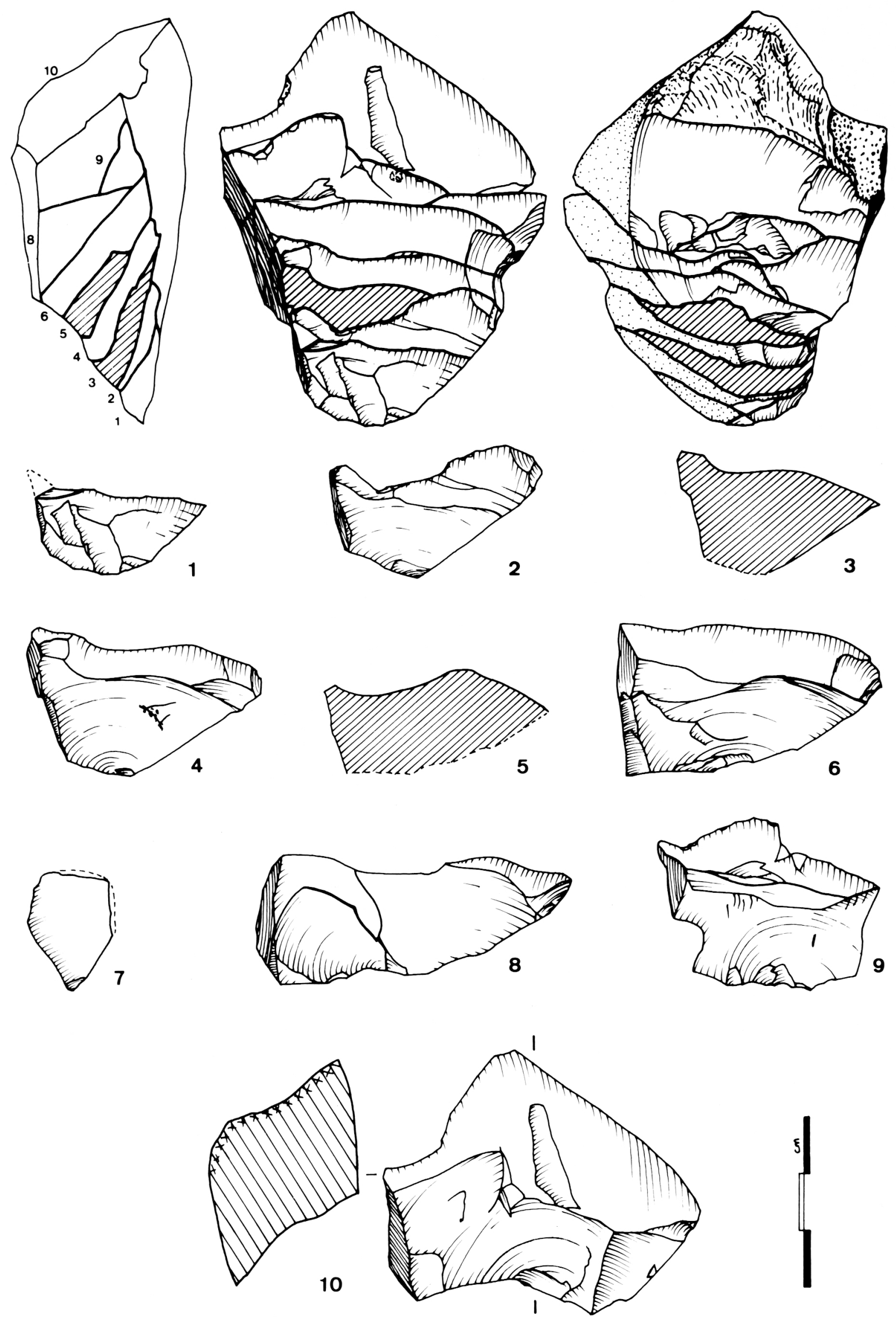

- Figure 4 : Le Pucheuil, niveau $6 e$ : remontage d'un ensemble d'éclats de type Le Pucheuil et du nucléus correspondant ; $n^{\circ} 1$ à 6 et $n^{\circ} 8$ et 9 : éclats de type Le Pucheuil (les $n^{\circ} 3$ et 5 , représentés par de fines hachures n'ont pas pu être remontés) ; $n^{\circ} 10$ : nucléus de type Le Pucheuil ; $n^{\circ} 7$ : petit éclat (renforçant la concavité proximale) 

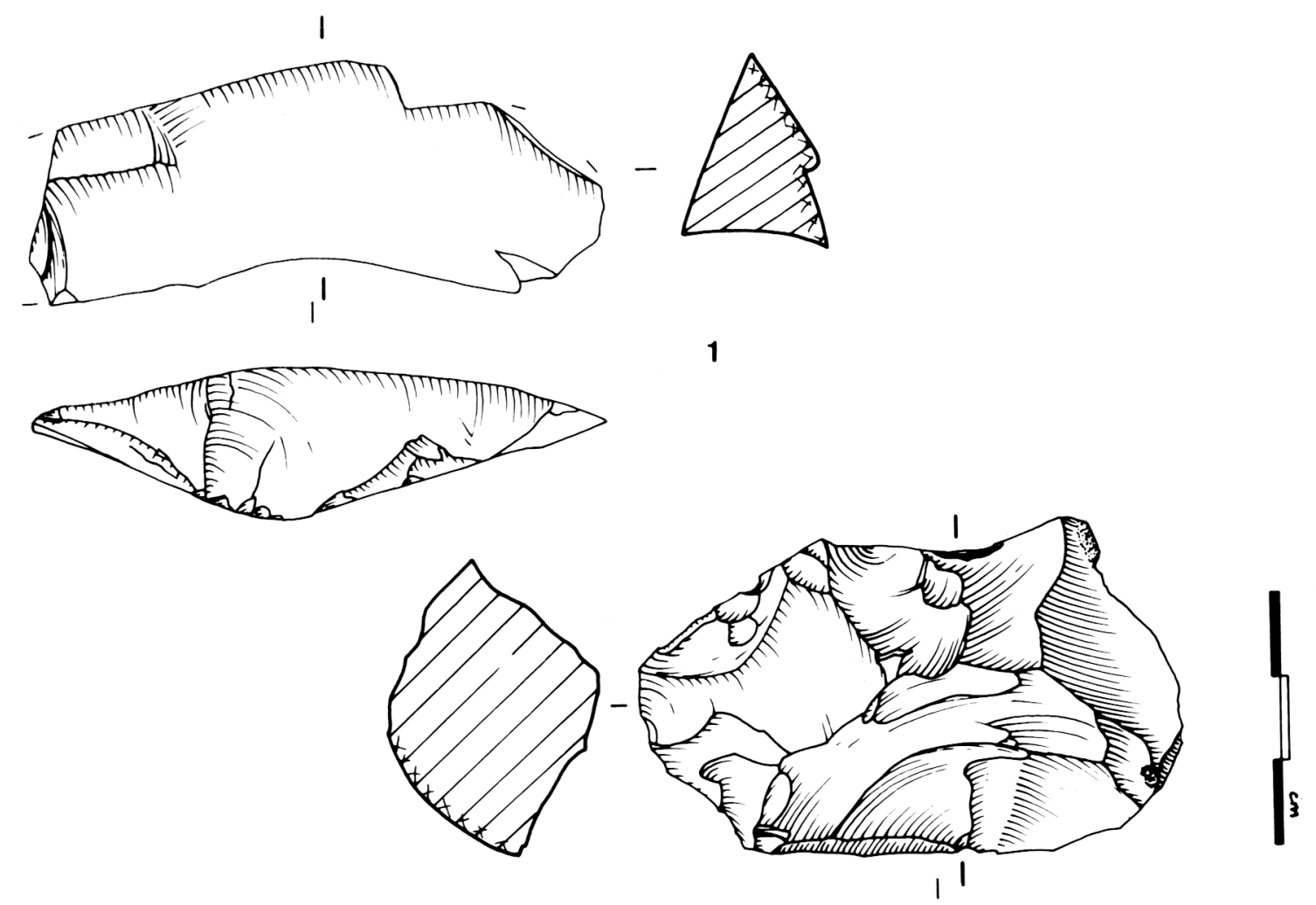

2

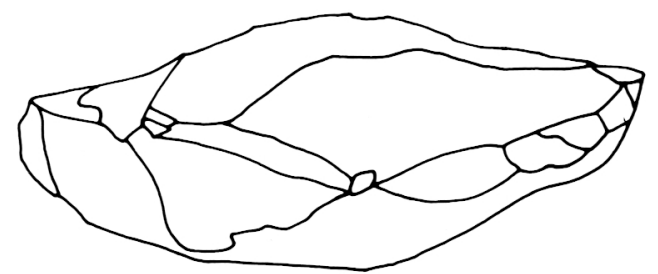

- Figure 5 : Le Pucheuil, niveau $6 e$ : nucléus de type Le Pucheuil réalisés à partir d'un éclat cortical cassé $\left(n^{\circ} 1\right)$ et d'un nucléus Levallois $\left(\mathrm{n}^{\circ} 2\right)$ 


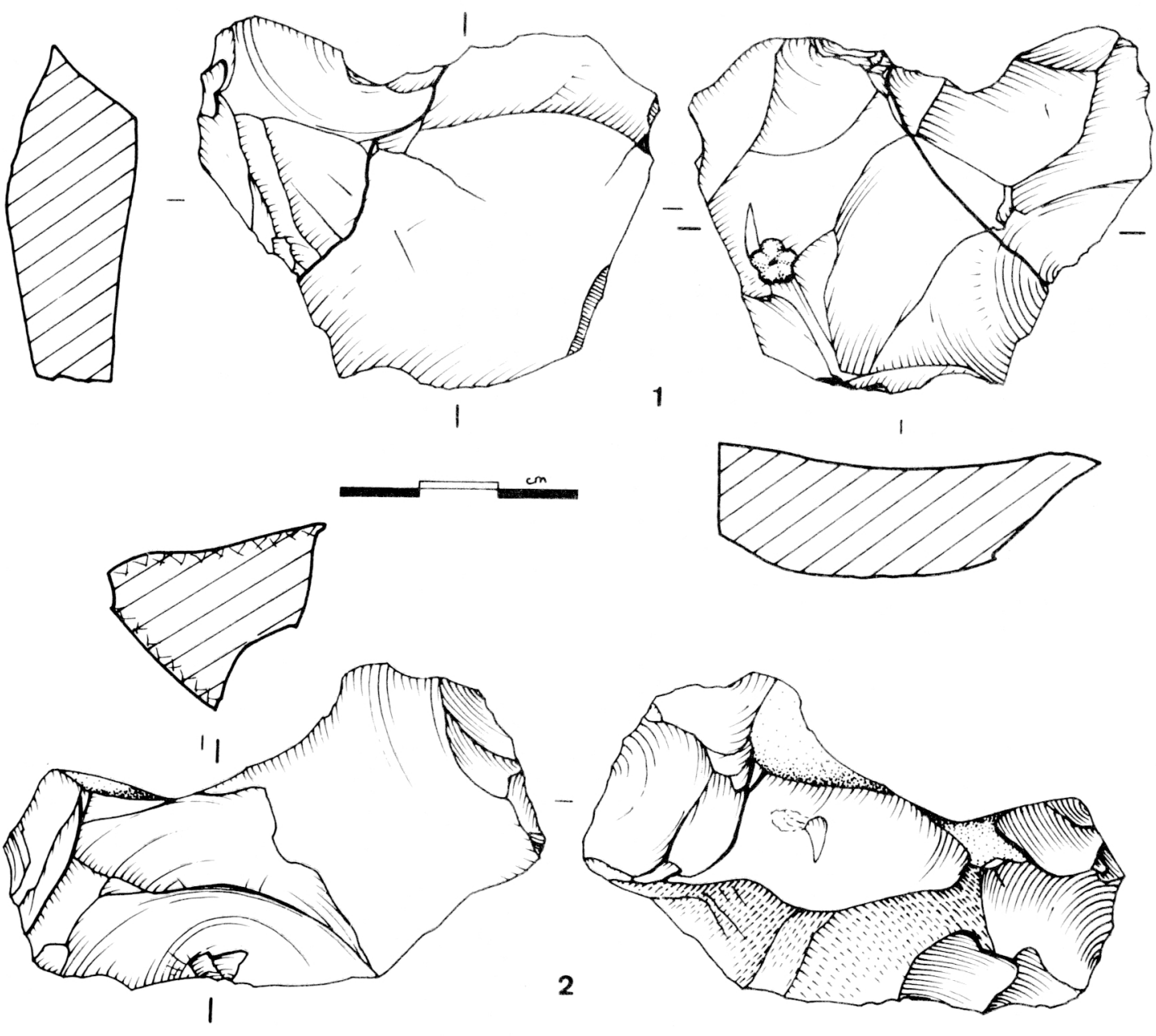

- Figure 6 : Le Pucheuil, niveau $6 \mathrm{e}$ : nucléus de type Le Pucheuil réalisés à partir d'un nucléus $\left(\mathrm{n}^{\circ} 1\right)$ et d'un éclat cortical $\left(n^{\circ} 2\right)$. 